\title{
A conditionally replicating adenovirus expressing IL-24 acts synergistically with temozolomide to enhance apoptosis in melanoma cells in vitro
}

\author{
ZHEN LIANG $^{1 *}$, CHUN-SHENG YANG ${ }^{2 *}$, FENG GU $^{3}$ and LAN-SHENG ZHANG ${ }^{4}$ \\ ${ }^{1}$ Department of Obstetrics and Gynecology, Xuzhou Central Hospital, Xuzhou, Jiangsu 221009; \\ ${ }^{2}$ Department of Dermatology, Affiliated Huai'an Hospital of Xuzhou Medical University, Huaian, \\ Jiangsu 223002; ${ }^{3}$ Clinical Laboratory, Xuzhou Tumor Hospital; ${ }^{4}$ Department of Oncology, The Second \\ Affiliated Hospital of Xuzhou Medical University, Xuzhou, Jiangsu 221002, P.R. China
}

Received July 16, 2015; Accepted December 13, 2016

DOI: $10.3892 / \mathrm{ol} .2017 .6007$

\begin{abstract}
Malignant melanoma is characterized by suppressed apoptosis in tumor cells and high levels of invasion. Temozolomide (TMZ) is one of the most effective single chemotherapeutic agents for patients with malignant melanoma, but resistance develops quickly and frequently. Therapeutic cytokines such as interleukin-24 (IL-24) and conditionally replicating adenoviruses have exhibited promising results as complementary therapies. Thus, the present study hypothesized that a conditionally replicating adenovirus expressing IL-24 combined with TMZ may exhibit increased antitumor activity compared with either treatment alone in melanoma A375 and M14 cell lines in vitro. The present study constructed an E1B-55 gene-deleted conditionally replicating adenovirus expressing the IL-24 gene (ZD55-IL-24). IL-24 was expressed at high levels in melanoma cells infected with ZD55-IL-24 in the presence of TMZ. The combination of ZD55-IL-24 + TMZ induced higher protein expression levels of the proapoptotic proteins B-cell lymphoma-2 (Bcl-2)-like protein 4 and phosphorylated protein, $\gamma-\mathrm{H} 2 \mathrm{~A}$ histone family member $\mathrm{X}(\gamma-\mathrm{H} 2 \mathrm{AX})$, and reduced the levels of the antiapoptotic proteins Bcl-2, myeloid cell leukemia-1and nuclear factor- $\kappa \mathrm{B}$ compared with either treatment individually. A dose-dependent increase in the cytopathic effects for the combination of ZD55-IL-24 and TMZ was also observed. The data of the present study suggest that the ZD55-IL-24 + TMZ combination induced increased levels of apoptosis, possibly by
\end{abstract}

Correspondence to: Dr Feng Gu, Clinical Laboratory, Xuzhou Tumor Hospital, 131 Huancheng Road, Xuzhou, Jiangsu 221002, P.R. China

E-mail: 53320023@qq.com

"Contributed equally

Key words: melanoma, conditionally replicating adenovirus, interleukin-24, temozolomide, apoptosis triggering DNA damage, in melanoma cells in vitro compared with either treatment alone. These findings suggest that this strategy may be a promising approach for the treatment of patients with malignant melanoma.

\section{Introduction}

Malignant melanoma is one of the most lethal and aggressive types of human malignancy, and is characterized by suppressed tumor cell apoptosis and high levels of invasion (1). Malignant melanoma is commonly treated with a combination of therapies including surgical removal, chemotherapy and radiotherapy. However, the reported long-term survival rate of melanoma remains low due to the ability of this malignancy to develop resistance to common chemotherapies and rapidly metastasize (2).

The alkylating agent temozolomide (TMZ) is one of the most effective single chemotherapeutic agents for patients with malignant melanoma (3), but resistance develops quickly and frequently (3). There has been relatively little progress in terms of developing novel pharmacological agents to treat metastatic melanoma over previous years. Cancer cells develop resistance to numerous pharmaceuticals through a variety of mechanisms, including decreased drug uptake, increased drug efflux, intracellular drug inactivation, repair of drug-induced damage and resistance to drug-induced apoptosis $(4,5)$. Gene therapy is one of the treatment strategies that have been proposed to circumvent drug resistance (6).

Interleukin-24 (IL-24) is a promising candidate for cancer gene therapy (7). A notable characteristic of IL-24 is that it preferentially inhibits growth and induces apoptosis in a variety of cancer cells without harming normal cells (8). Additionally, IL-24 exhibits potent 'anti-tumor bystander' activity, inhibits tumor angiogenesis, synergizes with chemotherapy, radiation and monoclonal antibody therapies, and exhibits immune modulatory activity (9). The synergistic effects of IL-24 and TMZ have been documented using a replication-defective adenovirus expressing IL-24 (Ad-IL-24) (10). However, replication-deficient adenoviruses such as Ad-IL-24 are generally considered inadequate to efficiently infect tumor cells (11). 
Conditionally replicating adenoviruses exhibit an advantage over replication-deficient adenovirus vectors and have been revealed to be effective for the treatment of malignant tumors (12). Conditionally replicating adenoviruses exhibit a dual mechanism of action in tumor cells, as they act as an oncolytic transgene delivery system that selectively replicates in and lyses tumor cells, and additionally amplify the expression and function of therapeutic genes in the tumor microenvironment $(13,14)$. Conditionally replicating adenoviruses may be generated by deleting a viral element that is necessary for virus replication in normal cells but is dispensable in tumor cells (15). An example of this approach is deleting the E1B-55 gene from the adenovirus ZD55 $(11,16)$. Conditionally replicating adenoviruses may also be used as potential vectors to express high levels of therapeutic genes in tumor cells (17). Therefore, the present study hypothesized that the combination of an E1B-55 gene-deleted conditionally replicating adenovirus expressing IL-24 and TMZ may synergistically provide an enhanced antitumor effect against malignant melanoma by inducing apoptosis.

\section{Materials and methods}

Cell culture and reagents. The human melanoma A375 and M14 cell lines were purchased from Nanjing KeyGen Biotech Co., Ltd. (Nanjing, China). HEK293 cells were purchased from Shanghai Cell Collection (Shanghai, China). The cells were cultured in Dulbecco's modified Eagle's medium (Gibco; Thermo Fisher Scientific, Inc., Waltham, MA, USA) supplemented with $10 \%$ heat-inactivated fetal bovine serum (Gibco; Thermo Fisher Scientific, Inc.), $4 \mathrm{mM}$ glutamine, $50 \mathrm{U} / \mathrm{ml}$ penicillin and $50 \mu \mathrm{g} / \mathrm{ml}$ streptomycin at $37^{\circ} \mathrm{C}$ in a humidified atmosphere with $5 \% \mathrm{CO}_{2}$. The cells were routinely screened to verify they were free of mycoplasma contamination, and were used for experiments in the logarithmic phase of growth.

TMZ, also known as 3,4-dihydro-3-methyl-4-oxoimidazo (5,1-d)-as-tetrazine-8-carboxamide (Schering-Plough Corporation, Kenilworth, NJ, USA) was dissolved in PBS and used at concentrations ranging between 0 and $800 \mu \mathrm{Mol} / 1$, as indicated. The experiments used 25, 50, 100, 200 and $400 \mu \mathrm{Mol} / \mathrm{l} \mathrm{TMZ}$.

Constructing the IL-24-expressing E1B-55-deleted adenovirus. The recombinant adenoviruses used in the present study have been previously described, which included ZD55 carrying IL-24 (ZD55-IL-24), ZD55 carrying enhanced green fluorescence protein (ZD55-EGFP), and adenovirus carrying IL-24 (Ad-IL-24) (18). The expression cassette containing the IL-24 gene under the control of the $\mathrm{H} 1$ promoter was produced by polymerase chain reaction (PCR) amplification of Ad-IL-24 and inserted into the pZD55 construct (18). The resulting product, pZD55-IL-24, lacked E1B-55 expression and produced IL-24. The plasmid, pZD55-IL-24, was co-transfected into HEK293 cells with the plasmid pBHGE3 (Microbix Biosystems, Toronto, Canada) to produce a recombinant conditionally replicating adenovirus, ZD55-IL-24.

The viral genome was extracted from the purified stock of ZD55-IL-24 using a QIAamp DNA blood mini kit (Qiagen GmbH, Hilden, Germany). The IL-24 gene was

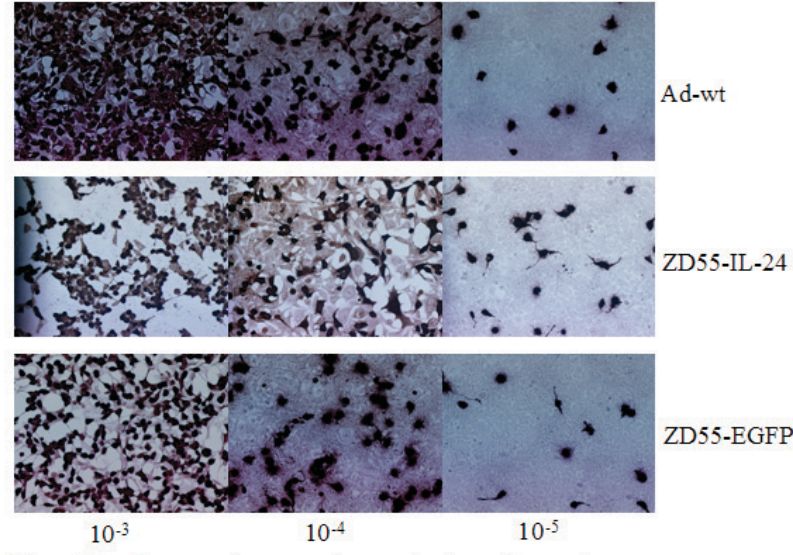

The titration analyses of oncolytic adenoviruses

\begin{tabular}{l|c}
\hline Adenoviruses & Virus titer $(\mathrm{pfu} / \mathrm{ml})$ \\
\hline Ad-wt & $2.5 \times 10^{10}$ \\
\hline ZD55-IL-24 & $4.0 \times 10^{10}$ \\
\hline ZD55-EGFP & $1.4 \times 10^{10}$ \\
\hline
\end{tabular}

Figure 1. Titration analyses of the two oncolytic adenoviruses (A) Representative images of plaque assays conducted in HEK293 cells using the Ad-wt, the IL-24-expressing E1B-55-deleted adenovirus ZD55-IL-24, and an EGFP-containing adenovirus lacking E1B-55, ZD55-EGFP. (B) Final virus titer in terms of pfu/ml. ZD55-IL-24, E1B-55 gene-deleted conditionally replicating adenovirus expressing the IL-24 gene; Ad-wt, wild type adenovirus; EGFP, enhanced green fluorescent protein.

verified by conventional PCR using the following primers: IL-24 forward, 5'-GAATTCGATATCTCTAGAC-3' and reverse, 5'-ATAGATATCTCAGAGCTTGTA-3'. The thermocycling conditions were $94^{\circ} \mathrm{C}$ for $5 \mathrm{~min}$, followed by 30 cycles at $94^{\circ} \mathrm{C}$ for $1 \mathrm{~min}, 50^{\circ} \mathrm{C}$ for $30 \mathrm{sec}, 72^{\circ} \mathrm{C}$ for $1 \mathrm{~min}$ and a final extension at $72^{\circ} \mathrm{C}$ for $10 \mathrm{~min}$. The amplification product was visualized by electrophoresis on a $1 \%$ agarose gel containing ethidium bromide. All viruses were plaque screened, propagated in HEK293 cells and purified by $\mathrm{CsCl}$ gradient ultracentrifugation following a previously described method (18). Viral concentration was determined by absorbance measurements at $260 \mathrm{~nm}$, and plaque-forming unit (pfu) titer was estimated by plaque assay as previously described (19) on HEK293 cells (Fig. 1). The PFU titer of ZD55-IL-24 was $4 \times 10^{10} \mathrm{PFU} / \mathrm{ml}$.

Western blot analysis. The cells were harvested from the plates and lysed in radioimmunoprecipitation (RIPA) buffer (pH 7.4) containing phenylmethylsulfonyl fluoride at a ratio of 100:1 (Beyotime Institute of Biotechnology, Haimen, China). Protein concentration was determined using a Bradford assay. The cell aliquots were separated using SDS-PAGE (12\% gel) with $50 \mu \mathrm{g}$ protein loaded/lane. The proteins were then transferred to nitrocellulose membranes and incubated overnight at $4^{\circ} \mathrm{C}$ with the primary antibodies (all 1:1,000 dilution). The following rabbit polyclonal primary antibodies were used: Anti-caspase-3 (cat. no. AB42437), anti-B-cell lymphoma-2 (Bcl-2; cat. no. AB40415), anti-myeloid cell leukemia-1 (Mcl-1; cat. no. AB38113), anti-Bcl-2-like protein 4 (Bax; cat. no. AB40636), anti-phosphorylated $\gamma$-H2AX (cat. no. AB41808), anti-nuclear factor (NF)-кB (cat. no. AB41808), 
A

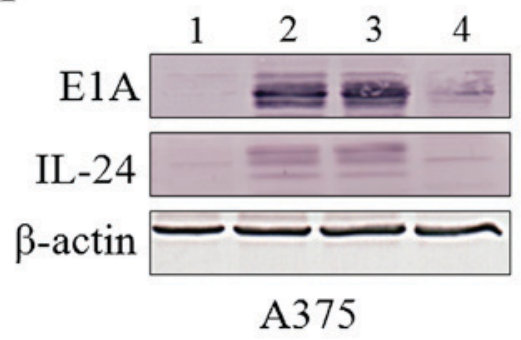

B

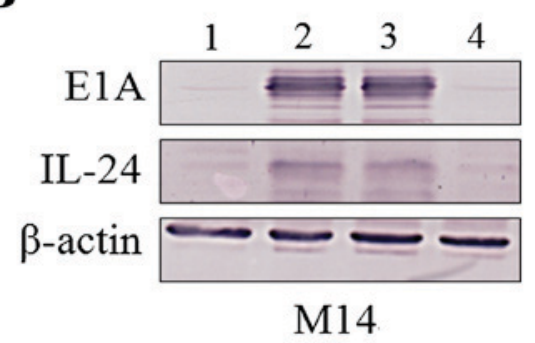

Figure 2. ZD55-IL-24 efficiently delivered IL-24 to (A) A375 and (B) M14 melanoma cells. Cells were infected with ZD55-IL-24 in the presence or absence of TMZ for $48 \mathrm{~h}$. Cells treated with PBS were used as the negative control. Levels of IL-24 and the adenovirus E1A protein were analyzed by western blotting. $\beta$-actin was used as the loading control. Lanes: 1, PBS; 2, ZD55-IL-24 only; 3, ZD55-IL-24 + TMZ; and 4, TMZ only.

anti-p53 (cat. no. AB38007) (all AbSci, Vancouver, WA, USA), anti-IL-24 (cat. no. bs-10576R; BIOSS, Beijing, China), anti- $\beta$-actin (cat. no. AP0731; Bioworld Technology, Inc., St. Louis Park, MN, USA), and anti-adenovirus early region 1A (E1A; cat. no. sc-374663; Santa Cruz Biotechnology, Inc., Dallas, TX, USA). The membranes were then washed with TBS-Tween 20 and incubated with goat anti-rabbit alkaline phosphatase-conjugated secondary antibodies (1:4,000 dilution, cat. no. ZB-2301; ZSGB-BIO, Zhongshan, Beijing) in TBS-Tween 20 at room temperature for $2 \mathrm{~h}$ and developed using a nitro-blue tetrazolium chloride/5-bromo-4-chloro-3'-indolyl phosphate p-toluidine salt color substrate (Promega Corporation, Madison, WI, USA). The changes in the expression levels of these proteins were determined using ImageQuant ${ }^{\mathrm{TM}}$ TL software (version 8.1; Molecular Dynamics; GE Healthcare Life Sciences, Chalfont, UK) and are expressed as a ratio relative to the protein level in PBS-treated cells, which was set at $100 \%$.

Cytopathic effect (CPE) assay. The cells were plated at a density of $1 \times 10^{5}$ cells in 96- or 48-well plates and treated with TMZ alone, ZD55-IL-24 alone or varying combinations of TMZ $(0,25,50,100,200$ and $400 \mu \mathrm{M})$ and ZD55-IL-24 [0, 1, 5 and 10, multiplicity of infection, (MOI)]. Following a 72- or 120-h culture at room temperature, the cells were washed with water, paraformaldehyde-fixed and stained with crystal violet (Amresco, LLC, Solon, OH, USA).

Statistical analysis. The data are expressed as the mean \pm standard deviation and were analyzed using an independent sample $t$-test and analysis of variance as appropriate. The analyses were performed using SPSS Base version 13.0 for Windows (SPSS, Inc., Chicago, IL, USA). P $<0.05$ was considered to indicate a statistically significant difference.

\section{Results}

ZD55-IL-24 efficiently delivers IL-24 into melanoma cells. A key component of the hypothesis of the present study was the inclusion of IL-24 in the combined antitumor therapy delivered by ZD55. Therefore, the present study first assessed whether ZD55-IL-24 was an efficient method of delivering IL-24 to melanoma tumor cells using the A375 and M14 cell lines. The cells were treated with ZD55-IL-24+TMZ,
A
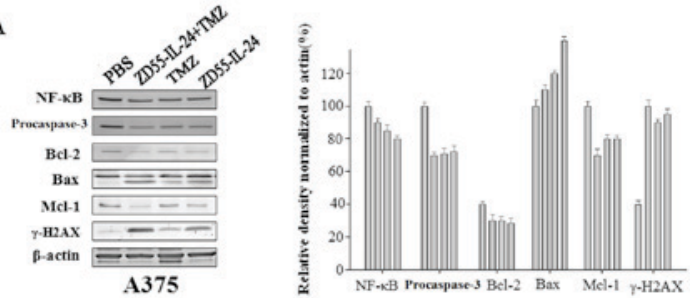

B
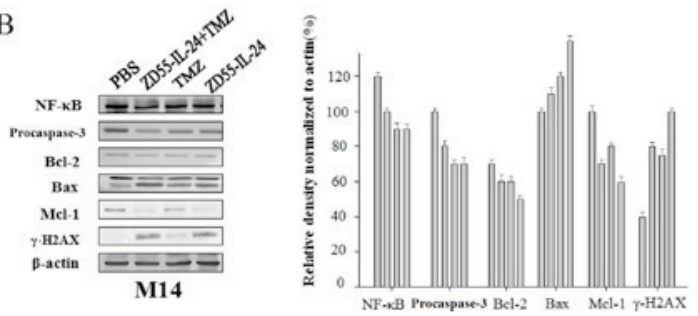

Figure 3. Combining ZD55-IL-24 with TMZ increased the expression of proteins associated with apoptosis induction. Melanoma cells (A) A375 and (B) M14 were treated with ZD55-IL-24 + TMZ, ZD55-IL-24 only or TMZ only. Cells treated with PBS were used as the negative control. The cells were lysed $72 \mathrm{~h}$ subsequent to treatment, and the protein expression levels of proapoptotic (Bax and $\gamma-\mathrm{H} 2 \mathrm{AX}$ ) and antiapoptotic (Bcl-2, Mcl-1 and $\mathrm{NF}-\kappa \mathrm{B})$ proteins were assessed. $\beta$-actin was used as the loading control. The change in protein expression level relative to the PBS control was quantified by densitometry. TMZ, temozolomide; IL, interleukin; ZD55-IL-24, E1B-55 gene-deleted conditionally replicating adenovirus expressing the IL-24 gene; Bcl-2, B-cell lymphoma-2; Bax, Bcl-2-like protein 4; $\mathrm{NF}-\kappa \mathrm{B}$, nuclear factor $\kappa \mathrm{B} ; \gamma-\mathrm{H} 2 \mathrm{AX}, \gamma-\mathrm{H} 2 \mathrm{~A}$ histone family member X; Mcl-1, myeloid cell leukemia-1.

ZD55-IL-24 alone or TMZ alone. The negative control was cells treated with PBS. The present study assessed the level of IL-24 protein by immunoblotting. IL-24 protein was clearly detectable XLVIII h subsequent to infection with ZD55-IL-24 in the A375 (Fig. 2A) and M14 (Fig. 2B) cells. No difference was observed in the level of IL-24 expression between the cells treated with ZD55-IL-24 alone or ZD55-IL-24 + TMZ (Fig. 2, lanes 2 and 3). These results demonstrated that ZD55 mediates the stable expression of high levels of IL-24 in the presence or absence of TMZ. The adenovirus protein E1A was used to assess whether there were differences in the level of infection in the presence of TMZ. Similar levels of E1A were detected in the presence or absence of TMZ, indicating that TMZ did not reduce the replication capability of the ZD55 adenovirus (Fig. 2). 

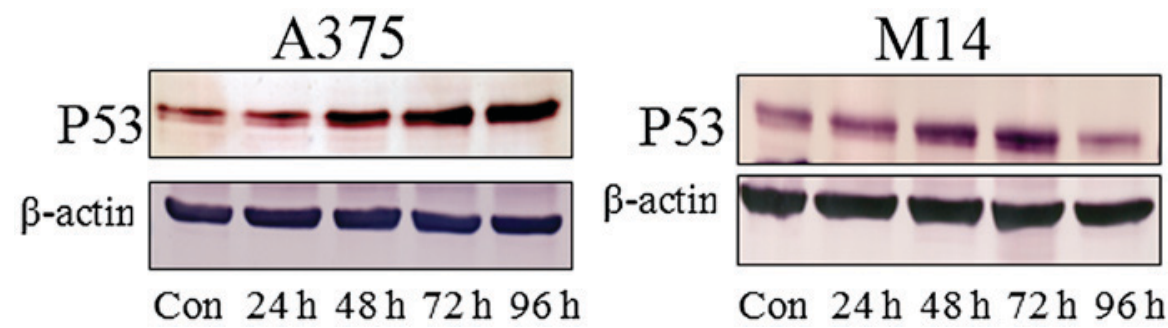

Figure 4. Combination of ZD55-IL-24 and TMZ upregulated p53 protein expression. Melanoma cells (A) A375 and (B) M14 were treated with ZD55-IL-24 + TMZ, ZD55-IL-24 only or TMZ only. The cells were lysed at the time points indicated and the p53 levels were analyzed by western blotting. $\beta$-actin was used as the loading control. TMZ, temozolomide; IL, interleukin; ZD55-IL-24, E1B-55 gene-deleted conditionally replicating adenovirus expressing the IL-24 gene; Con, control

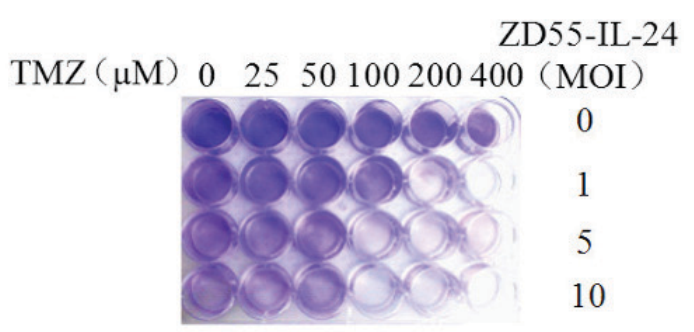

A375

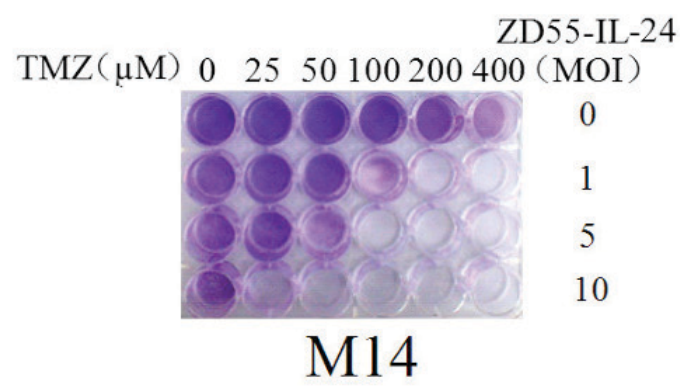

Figure 5. Combination of ZD55-IL-24 and TMZ increased the CPE of each treatment. Melanoma (A) A375 and (B) M14 cells were treated with different concentrations/doses of ZD55-IL-24 and TMZ in a systematic matrix to assess the CPE in melanoma cells. Cells were plated at a density of $10^{5}$ cells/6-cm dish and stained with crystal violet 7 days subsequent to infection. The presence of staining indicated live cells. Representative results of a CPE assay in A375 and M14 cells are shown. TMZ, temozolomide; IL, interleukin; ZD55-IL-24, E1B-55 gene-deleted conditionally replicating adenovirus expressing the IL-24 gene; $\mathrm{CPE}$, cytopathic effect; MOI, multiplicity of infection.

Combining ZD55-IL-24 with TMZ increases the expression of proteins associated with apoptosis induction. To elucidate the molecular mechanism through which ZD55-IL-24 and TMZ induced apoptosis, the present study examined the protein expression levels of $\mathrm{NF}-\kappa \mathrm{B}, \mathrm{Bcl}-2, \mathrm{Mcl}-1$, Bax and caspase-3 subsequent to infection with ZD55-IL-24, with or without TMZ. The marker $\gamma$-H2AX was also included as a marker of DNA damage, a potential apoptotic trigger. The A375 and M14 cells were infected with ZD55-IL-24 in the presence or absence of TMZ, and the protein levels of Mcl-1, Bcl-2, Bax, NF- $\kappa \mathrm{B}, \gamma-\mathrm{H} 2 \mathrm{AX}$ and caspase-3 were determined by immunoblotting. PBS-treated cells were used as the negative control. Representative western blot images from A375 (Fig. 3A) and M14 (Fig. 3B) cells are shown. The present study also quantified the protein expression using densitometry, and expressed the change in protein expression as a ratio of the protein expression level in PBS-treated cells (which was considered to be $100 \%$ ). ZD55-IL-24 alone reduced the protein expression levels of Mcl-1, $\mathrm{Bcl}-2$ and $\mathrm{NF}-\kappa \mathrm{B}$. The addition of TMZ additionally reduced the protein expression levels of the above proteins. In contrast, ZD55-IL-24 + TMZ significantly increased the protein expression levels of Bax and $\gamma-\mathrm{H} 2 \mathrm{AX}$. TMZ alone did not significantly increase Bax expression (ZD55-IL-24 + TMZ; Fig. 3).

ZD55-IL-24 and TMZ upregulate p53 expression. The present study then investigated whether ZD55-IL-24 regulates the protein expression levels of p53. A375 and M14 cells were infected with ZD55-IL-24 in the presence or absence of TMZ, and the expresion of p53 protein was assessed at 24-96 h post-infection. ZD55-IL-24 treatment increased the protein level of p53 (Fig. 4). By contrast, TMZ induced weak p53 expression. The combination of ZD55-IL-24+TMZ increased the level of p53 in a time-dependent manner.

Combination of ZD55-IL-24 and TMZ exhibits potent CPEs. Having revealed that proteins associated with the induction of apoptosis were upregulated by ZD55-IL-24 and TMZ, the present study then assessed the CPE of the combined treatment. The CPE of the combined treatment was evaluated across a concentration gradient of TMZ $(\mu \mathrm{M})$ and a range of infecting doses of ZD55-IL-24 (MOI) in a systematic matrix (Fig. 5). The cells were stained with crystal violet 7 days subsequent to infection. As shown in Fig. 5, the CPEs of ZD-55-IL-24 + $\mathrm{TMZ}$ were dose dependent. Each concentration of TMZ tested, including ZD55-IL-24, produced greater CPE compared with that of TMZ alone in a dose-dependent manner. Similarly, each ZD55-IL-24 MOI tested including TMZ increased the CPE in a dose-dependent manner. These results clearly supported the hypothesis that the combination of TMZ and ZD55-IL-24 exhibits an increased antitumor activity compared with that caused by either treatment alone.

\section{Discussion}

As conditionally replicating adenoviruses may act as antitumor agents and delivery vectors for therapeutic genes, the present study evaluated whether a conditionally replicating adenovirus lacking the E1B-55 gene and expressing IL-24 may act synergistically with TMZ to destroy melanoma cells via apoptosis.

The induction of apoptosis in tumor cells is an important mechanism of cytotoxicity for the majority of anticancer 
therapies (20). The present study revealed that ZD55-IL-24 + TMZ decreased the levels of NF- $\mathrm{NB}, \mathrm{Bcl}-2$ and $\mathrm{Mcl}-1$, but increased the levels of Bax and $\gamma-\mathrm{H} 2 \mathrm{AX}$, and induced a marked activation of caspase-3. The overall change in protein expression levels suggested that ZD55-IL-24 combined with TMZ changed the ratio of proapoptotic to antiapoptotic proteins in melanoma cells, which may have contributed to the induction of apoptosis, potentially through damaging the tumor cell DNA.

$\mathrm{NF}-\kappa \mathrm{B}$ activation exhibits an antiapoptotic role, and promotes cell survival and growth (21). Chemotherapeutic agents and therapeutic cytokines prevent the prolonged activation of the NF- $\kappa$ B protein (22). The combination of ZD55-IL-24 + TMZ inhibited NF- $\mathrm{B}$ protein expression compared with the negative control and either treatment alone in the melanoma A375 and M14 cells.

In conclusion, the combination of ZD-55-IL-24 and TMZ was more effective at destroying melanoma cells in vitro than either treatment alone. Thus, the strategy of combining conditionally replicating adenoviruses, therapeutic cytokines and chemotherapy appears to be a promising candidate for the treatment of patients with malignant melanoma.

\section{References}

1. La Porta CA: Mechanism of drug sensitivity and resistance in melanoma. Curr Cancer Drug Targets 9: 391-397, 2009.

2. Nath K, Nelson DS, Ho AM, Lee SC, Darpolor MM, Pickup S, Zhou R, Heitjan DF, Leeper DB and Glickson JD: (31) P and (1) H MRS of DB-1 melanoma xenografts: Lonidamine selectively decreases tumor intracellular $\mathrm{pH}$ and energy status and sensitizes tumors to melphalan. NMR Biomed 26: 98-105, 2013.

3. Jiang G, Li RH, Sun C, Jia HY, Lei TC and Liu YQ: Efficacy and safety between temozolomide alone and temozolomide-based double therapy for malignant melanoma: A meta-analysis. Tumour Biol 35: 315-322, 2014.

4. Plummer ER, Middleton MR, Jones C, Olsen A, Hickson I, McHugh P, Margison GP, McGown G, Thorncroft M, Watson AJ, et al: Temozolomide pharmacodynamics in patients with metastatic melanoma: DNA damage and activity of repair enzymes O6-alkylguanine alkyltransferase and poly(ADP-ribose) polymerase-1. Clin Cancer Res 11: 3402-3409, 2005.

5. Jiang G, Li LT, Xin Y, Zhang L, Liu YQ and Zheng JN: Strategy for reversing resistance to temozolomide in malignant melanoma Curr Med Chem 19: 3886-3892, 2012.

6. Jiang G, Liu YQ, Wei ZP, Pei DS, Mao LJ and Zheng JN: Enhanced anti-tumor activity by the combination of a conditionally replicating adenovirus mediated interleukin-24 and dacarbazine against melanoma cells via induction of apoptosis. Cancer Lett 294: 220-228, 2010.

7. Tian H, Zhang DF, Zhang BF, Li HZ, Zhang Q, Li LT, Pei DS and Zheng JN: Melanoma differentiation associated gene-7/interleukin-24 induces caspase-3 denitrosylation to facilitate the activation of cancer cell apoptosis. J Interferon Cytokine Res 35 157-167, 2015.
8. Dent P, Yacoub A, Hamed HA, Park MA, Dash R, Bhutia SK, Sarkar D, Wang XY, Gupta P, Emdad L, et al: The development of MDA-7/IL-24 as a cancer therapeutic. Pharmacol Ther 128: 375-384, 2010

9. Emdad L, Lebedeva IV, Su ZZ, Gupta P, Sauane M, Dash R, Grant S, Dent P, Curiel DT, Sarkar D and Fisher PB: Historical perspective and recent insights into our understanding of the molecular and biochemical basis of the antitumor properties of mda-7/IL-24. Cancer Biol Ther 8: 391-400, 2009.

10. Zheng M, Bocangel D, Ramesh R, Ekmekcioglu S, Poindexter N, Grimm EA and Chada S: Interleukin-24 overcomes temozolomide resistance and enhances cell death by down-regulation of O6-methylguanine-DNA methyltransferase in human melanoma cells. Mol Cancer Ther 7: 3842-3851, 2008.

11. Liu XY: Targeting gene-virotherapy of cancer and its prosperity. Cell Res 16: 879-886, 2006.

12. Liu XY, Li HG, Zhang KJ and Gu JF: Strategy of Cancer Targeting Gene-Viro-Therapy (CTGVT) a trend in both cancer gene therapy and cancer virotherapy. Curr Pharm Biotechnol 13: 1761-1767, 2012.

13. Zhao L, Gu J, Dong A, Zhang Y, Zhong L, He L, Wang Y, Zhang J, Zhang Z, Huiwang J, et al: Potent antitumor activity of oncolytic adenovirus expressing mda-7/IL-24 for colorectal cancer. Hum Gene Ther 16: 845-858, 2005.

14. Zhang ZY, Wang LQ, Fu CF, Li X, Cui ZL, Zhang JY, Xue SH, Sun $\mathrm{N}$ and $\mathrm{Xu} F$ : Combination of targeting gene-viro therapy with recombinant Fowl-pox viruses with HN and VP3 genes on mouse osteosarcoma. Eur Rev Med Pharmacol Sci 17: 767-776, 2013.

15. Jiang G, Jiang AJ, Cheng Q, Tian H, Li LT and Zheng JN: A dual-regulated oncolytic adenovirus expressing interleukin-24 sensitizes melanoma cells to temozolomide via the induction of apoptosis. Tumour Biol 34: 1263-1271, 2013.

16. Zhou X, Xie G, Wang S, Wang Y, Zhang K, Zheng S, Chu L, Xiao L, Yu Y, Zhang Y and Liu X: Potent and specific antitumor effect for colorectal cancer by $\mathrm{CEA}$ and $\mathrm{Rb}$ double regulated oncolytic adenovirus harboring ST13 gene. PLos One 7: e47566, 2012.

17. Jiang G, Zhang L, Xin Y, Pei DS, Wei ZP, Liu YQ and Zheng JN: Conditionally replicating adenoviruses carrying mda-7/IL-24 for cancer therapy. Acta Oncol 51: 285-292, 2012.

18. Zhao L, Dong A, Gu J, Liu Z, Zhang Y, Zhang W, Wang Y, He L, Qian C, Qian Q and Liu X: The antitumor activity of TRAIL and IL-24 with replicating oncolytic adenovirus in colorectal cancer. Cancer Gene Ther 13: 1011-1022, 2006.

19. Qiu S, Ruan H, Pei Z, Hu B, Lan P, Wang J, Zhang Z, Gu J, Sun L, Qian C, et al: Combination of targeting gene-virotherapy with 5-FU enhances antitumor efficacy in malignant colorectal carcinoma. J Interferon Cytokine Res 24: 219-230, 2004.

20. Berger A, Quast SA, Plötz M, Hein M, Kunz M, Langer P and Eberle J: Sensitization of melanoma cells for death ligand-induced apoptosis by an indirubin derivative-Enhancement of both extrinsic and intrinsic apoptosis pathways. Biochem Pharmacol 81: 71-81, 2011.

21. Kong XJ, Duan LJ, Qian XQ, Xu D, Liu HL, Zhu YJ and Qi J: Tumor-suppressive microRNA-497 targets IKK $\beta$ to regulate $\mathrm{NF}-\kappa \mathrm{B}$ signaling pathway in human prostate cancer cells. Am J Cancer Res 5: 1795-1804, 2015.

22. Wilczynski J, Duechler M and Czyz M: Targeting NF-кB and HIF-1 pathways for the treatment of cancer: Part I. Arch Immunol Ther Exp (Warsz) 59: 289-299, 2011. 